\title{
Ist der Zusatz von Kortison sinnvoll?
}

Fragestellung: Diese Studie untersucht, ob eine sechswöchige, adjuvante Therapie mit Dexamethason zu einem verbesserten Outcome bei HIV-Patienten mit einer akuten Kryptokokkenmeningitis führt.

Hintergrund: Die Kryptokokkenmeningitis ist nach wie vor einer der häufigsten opportunistischen Infektionen bei Patienten mit einer schlecht kontrollierten HIV-Infektion. Die weltweite jährliche Mortalität wird auf 600.000 geschätzt $[1,2]$. Die Therapie hat sich dabei über die letzten 20 Jahre mangels neuerer, gegen Kryptokokken wirksamer, antifungaler Medikamente nicht geändert. Somit besteht die Standardtherapie immer noch in Amphotericin B und Fluconazol.

Patienten und Methodik: In der randomisierten, placebokontrollierten Doppelblindstudie erhielten die Patienten die Standardtherapie mit Amphoteri-

Beardsley J, Wolbers M, Kibengo FM et al; CryptoDex Investigators. Adjunctive dexamethasone in HIV-associated cryptococcal meningitis. N Engl J Med 2016; 374: $542-54$ cin B und Fluconazol und zusätzlich über sechs Wochen Dexamethason oder Placebo. Die Studie wurde in Asien (Thailand, Vietnam, Laos, Indonesien) und Afrika (Malawi, Uganda) durchgeführt.
Das Dexamethason wurde in der ersten Woche mit $0,3 \mathrm{mg} / \mathrm{kg}$ KG i.v., in der zweiten Woche mit $0,2 \mathrm{mg} / \mathrm{kg} \mathrm{KG}$ i.v., in der dritten Woche mit $0,1 \mathrm{mg} / \mathrm{kg} \mathrm{KG} \mathrm{p.o.,} \mathrm{in} \mathrm{der} \mathrm{vierten} \mathrm{Woche} \mathrm{mit} 3$ $\mathrm{mg} / \mathrm{Tag}$ p.o., in der fünften Woche mit $2 \mathrm{mg} / \mathrm{Tag}$ p.o. und in der sechsten Woche mit $1 \mathrm{mg} /$ Tag p. o. verabreicht. Der primäre Outcome Parameter sollte das Überleben zehn Wochen nach Randomisierung sein.

Ergebnisse: Die Studie wurde aus Sicherheitsgründen vorzeitig nach 451 eingeschlossenen Patienten gestoppt. Sowohl die Mortalität als auch der Grad der Behinderung waren unter Dexamethason höher als unter Placebo. Nach Woche 10 waren $47 \%$ versus $41 \%$ und nach sechs Monaten $57 \%$ versus $49 \%$ der Patienten in der Dexamethason- versus Placebogruppe verstorben. Die Kryptokokken-Clearence im Liquor war deutlich langsamer in der Dexamethason-Gruppe. Zudem waren kardiale und renale Nebenwirkungen wie auch schwere Infektionen signifikant häufiger in dieser Gruppe. Die Ergebnisse waren konkordant zwischen den afrikanischen und asiatischen Studienzentren.

Schlussfolgerungen: Die adjuvante Behandlung mit Dexamethason führt nicht zu einer Verminderung der Mortalität oder zu einem besseren Outcome bei HIV-Patienten mit einer Kryptokokkenmeningitis.

\section{- Kommentar von Matthias Maschke, Trier}

\section{Kortikosteroide können nicht empfohlen werden}

Der Einsatz von Kortikosteroiden führt bei Patienten mit Pneumokokkenmeningitis und tuberkulöser Meningitis zu einer Verbesserung des Outcomes. Insofern war die Annahme, dass sie auch einen Effekt bei der Kryptokokkenmeningitis haben könnten, insbesondere vor dem Hintergrund des häufig erhöhten Hirndrucks und kryptokokkenbedingter Vaskulitiden, plausibel. Zudem wäre es möglich, dass ein kryptokokkenassoziiertes Immunrekonstitutionssyndrom durch Dexamethason vermieden wird. Letztlich war das Ergebnis der Studie aber eindeutig negativ, sodass der Einsatz von Kortikosteroiden bei einer Kryptokokkenmeningitis nicht empfohlen werden kann. Zu beachten ist allerdings, dass die eingeschlossenen HIV-Patienten zu 60 - 77\% keine antiretrovirale Medikation erhielten und die CD4+-Zellzahl mit einem Median von 18/ul sehr niedrig war, was die hohe Gesamtmortalität der Patienten mit erklären dürfte. Aufgrund der mittlerweile in Westeuropa und Nordamerika durch die moderne antiretrovirale Therapie (cART) bedingte geringe Inzidenz von Kryptokokkenmeningitiden wird es aber wahrscheinlich keine Studie zum Einsatz von Kortikosteroiden bei einem europäischen oder amerikanischen Patientenkollektiv geben [3].

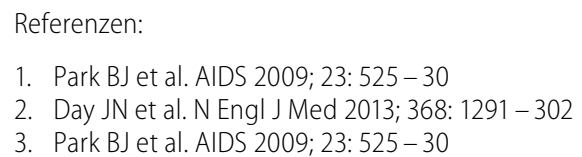

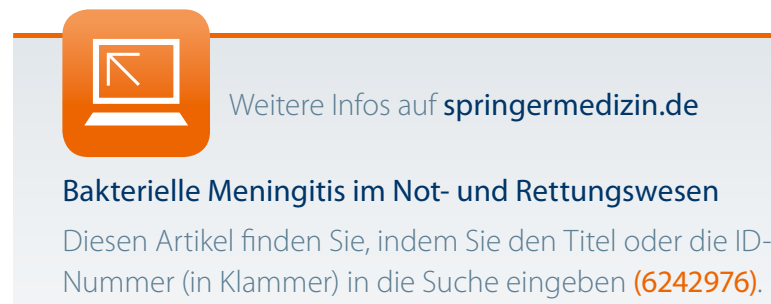

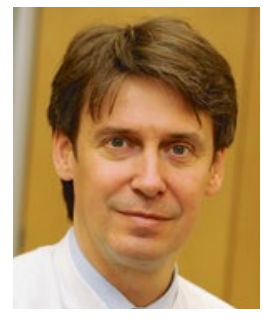

Prof. Dr. med. Matthias Maschke, Trier

Chefarzt der Abteilung für Neurologie und Neurophysiologie, Krankenhaus der Barmherzigen Brüder Trier E-Mail: m.maschke@bk-trier.de 